\title{
P.I.ZABOLOTNYI
}

\section{MODEL OF E-POLARIZED WAVE PROPAGATION IN A MULTILAYER DIELECTRIC STRUCTURE}

\author{
Institute of Technical Mechanics \\ of the National Academy of Sciences of U kraine and the State Space Agency of U kraine \\ 15 Leshko-Popelya St., D nipro 49005, U kraine; e-mail: mwave@ ukr.net
}

\begin{abstract}
This paper addresses the determination of the dielectric constant of multilayer dielectric structures by radiowave interferometry. In the general case, in interferometry measurements to one measured value of the reflection coefficient there may correspond an infinity of dielectric constants. This ambiguity may be resolved by first determining the effect of different parameters of the probing electromagnetic wave on the reflection coefficient. In particular, it is important to have a preliminary estimate of the effect of the incidence angle and the polarization on the range of variation of the reflection coefficient with the variation of one of the structure parameters.

This paper considers the case where a plane E-polarized electromagnetic wave, i.e. a wave whose magnetic field is perpendicular to the incidence plane, is incident on a multilayer dielectric structure. The aim of this work is to develop a model of the propagation of an E-polarized electromagnetic wave through a multilayer dielectric structure at an arbitrary incidence angle and to determine the range of variation of the reflection coefficient with the variation of the dielectric constants of the layers. The paper presents a model of the propagation of an Epolarized electromagnetic wave in a two-layer dielectric structure. A metal base, which is an ideal conductor, underlies the structure. The electromagnetic wave is incident from the air at an arbitrary incidence angle. Based on the model, a method is proposed for measuring the relative dielectric constant and the dielectric loss tangent. It is shown that at a normal incidence the reflection coefficient magnitude is the same both for $\mathrm{H}$ - and E-polarization. Because of this, determining the relative dielectric constant and the loss tangent from the measured reflection coefficient magnitude calls for measurements not only at a normal incidence, but also at an oblique incidence, at which the reflection coefficient magnitudes will be different for $\mathrm{H}$ - and E-polarization.
\end{abstract}

Keywords multilayer dielectric structures, E-polarization, $\mathrm{H}$-polarization, dielectric constant, reflection coefficient.

1. Borul'ko V. F., Drobakhin O. O., Slavin I. V. Multifrequency Nondestructive Microwave Methods for the Characterization of Layered Dielectrics.

Dnipropetrovsk: Dnipropetrovsk State University, 1992. 120 pp. (in Russian).

2. Shaarawi A. M., Besieris I. M., Attiya A. M., El-Diwan E. Reflection and transmission of an electromagnetic $X$-wave incident on a planar air-dielectric interface: spectral analysis. Progress in Electromagnetics Research. 2001. V. 30. Pp. 213-249.

https://doi.org/10.2528/PIER00042502

3. Kaz'min A. I., Fedyunin P. A. Reconstruction of the structure of the electrophysical parameters of multilayer dielectric materials and coatings from the frequency dependence of the attenuation coefficient of the field of a surface electromagnetic wave. Measurement Techniques. 2019. V. 62. Pp. 809-816.

https://doi.org/10.1007/s11018-019-01699-7

4. Lytvynenko L. M., Prosvirnin S. L. Wave Diffraction by Periodic Multilayer Structures. Cottenham. UK: Cambridge Scientific Publishers, 2012. 158 pp.

5. Gerekos C., Tamponi A., Carrer L., Castelletti D., Santoni M., Bruzzone L. A Coherent Multilayer Simulator of Radargrams Acquired by Radar Sounder Instruments. IEEE Trans. on Geoscience and Remote Sensing. 2018, V. 56. 
Iss. 12. Pp. 7388-7404.

https://doi.org/10.1109/TGRS.2018.2851020

6. Kaliberda M. E., Lytvynenko L. M., Pogarsky S. A. The h-polarized electromagnetic wave diffraction by multi-element plane semi-infinite grating. Radio Physics and Radio Astronomy. 2014. V. 19. No. 4. Pp. 348-357 (in Russian).

https://doi.org/10.15407/rpra19.04.348

7. Chen L. F, Ong C. K., Neo C. P. , Varadan V. V., Varadan V. K. Microwave Electronics: Measurement and Materials Characterization. New York: John Wiley \& Sons, Ltd, 2004. 537 pp.

https://doi.org/10.1002/0470020466

8. Drobakhin O. O., Plaksin S. V., Riabchii V. D., Saltykov D. Yu. Engineering and Microwave Semiconductor Electronics. Dnipro: Dnipro National University. 335 pp. (in Ukrainian).

9. Zabolotnyi P.I. Model of $\mathrm{H}$-polarized wave propagation in the multilayer dielectric structure. Teh. Meh. 2021. No 1. Pp. 84-91.

https://doi.org/10.15407/itm2021.01.084 